\title{
Repetition between and within modalities in free recall*
}

\author{
J. ELISABETH WELLS \\ Massey University, Palmerston North, New Zealand \\ and \\ K. KIRSNER \\ University of Western Australia, Nedlands, Western Australia
}

\begin{abstract}
A repetition experiment was designed to test the effects of lag and modality changes on free recall. The results showed a powerful lag effect over the range of 0-9 items, however, there were no differences in recall between items repeated in the same or different modality in the 56-item list. Either the encoding variability hypothesis is wrong or it applies only to variation in semantic attributes, not to variation in physical attributes such as modality.
\end{abstract}

In free recall, there is considerable evidence that distributed repetitions result in higher recall than massed repetitions (Melton, 1970). Furthermore, in most of these studies recall increases monotonically with lag, that is, the spacing between repetitions. There are several explanations of these spacing effects and so far, it has proved difficult to distinguish between them. Bjork and Allen (1970) have effectively refuted any consolidation hypothesis but this still leaves a variety of encoding variability models (e.g., Madigan, 1969; Melton, 1970; Bower, 1972) and differential processing models (e.g., Rundus, 1971; Glanzer \& Duarte, 1971).

The encoding variability hypothesis states that, as lag increases, the two presentations of the same nominal item are more likely to be encoded differentially. In free recall, the two presentations may be encoded in different contexts or subjective units, thus increasing the number of retrieval cues for the item. Differential processing models assume that an item recognized as having already occurred will be processed less than one perceived as a new item. This may be because an item will not enter the short-term store if a trace of that item is still in the store (Glanzer \& Duarte, 1971) or because rehearsal patterns are affected by repetitions (Rundus, 1971).

All of these explanations imply that the effects of spacing are due to the resultant differences in the processing or encoding of repeated items, not to lag per se. Therefore, other means of causing differences should have the same effect as lag; that is, variant repetitions should be recalled better than nominally identical repeititons.

*This research was supported by Grant APA 146 from the National Research Council of Canada and OMHF Grant 164 from the Ontario Mental Health Foundation while both authors were working with B. B. Murdock, Jr., at the University of Toronto, Toronto, Ontario, Canada. This paper is sponsored by B. B. Murdock, Jr., who takes full editorial responsibility for its contents.
This prediction has been confirmed in studies using some kind of semantic variation. For example, by changing the language (Glanzer \& Duarte, 1971), by embedding words in different sentences (D'Agostino \& De Remer, 1973), or by modifying nouns with different adjectives (Bevan \& Dukes, 1967). However, the effects of lag and variation do not seem to be additive; studies investigating both lag and semantic variation (D’Agostino \& De Remer, 1973; McCormack \& Carboni, 1973; and Glanzer \& Duarte, 1971) have found semantic variation to be most beneficial at short lags.

It seems then, that semantic variation leads to better recall. Does physical or nonsemantic variation produce the same effect? Madigan and Doherty (1971) found no significant advantage for items repeated in different modalities rather than the same modality, in spite of their within-S design and the finding that Ss could remember whether presentations were visual or auditory. However, Madigan and Doherty (1971) used a median lag of eight items between repetitions and did not systematically vary lag; perhaps their results concealed advantages for different repetitions at short lags? The experiment reported in this paper investigated both modality of repetition and lag. A within-S, within-list design was used for both factors. Thus, the experiment replicates the major design features of Glanzer and Duarte's (1971) experiment on repetition within or across languages.

\section{METHOD}

\section{Design}

For repeated items there were four modality repetition conditions, auditory-auditory (AA), auditory-visual (AV), visual-auditory (VA) and visual-visual (VV), combined with four lag conditions $(0,1,3$, or 9 intervening items). Thus, the design was a 2 by 2 by 4 within-Ss design, the factors being modality of first presentation, modality of second presentation, and lag. The between-S factors concerned only the particular sets of lists used. 


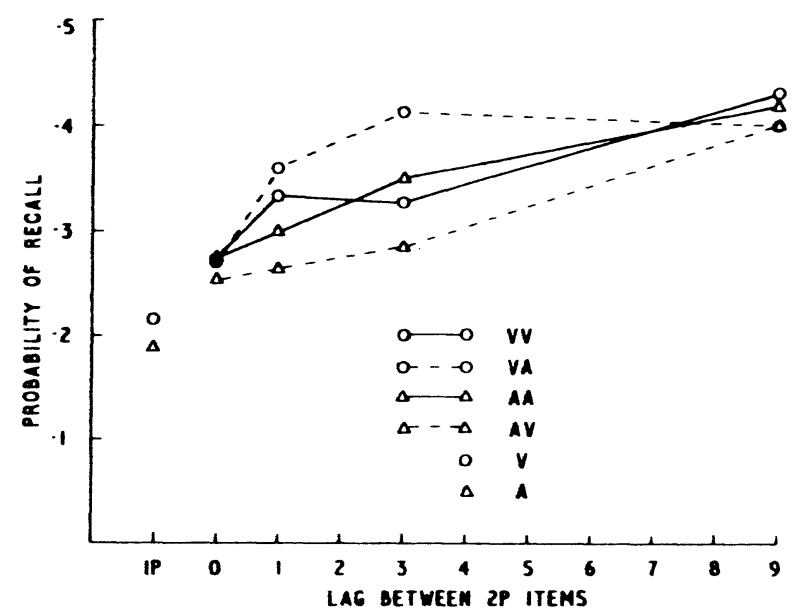

Fig. 1. Recall of single items (1P) and repeated items (2P) from the middle section of the lists (408 observations per point except for 1P items, which are based on 1,632 observations).

\section{Lists}

Each list was 56 words long and contained 36 different words. The middle 40-item section of the list contained 8 once-presented words, 4 visual and 4 auditory, and 16 repeated words, one for each of the $16 \mathrm{Lag}$ by Modality repetition conditions. The 8-item primacy and recency buffers each contained 4 once-presented words, 2 visual and 2 auditory, and 2 repeated words. Summing over the set of 12 lists given to any $\mathrm{S}$ resulted in three observations per modality for once-presented words at each serial position in the buffers. The words in the lists were from the Toronto Word Pool.

There were four sets of 12 lists made up from two sets of within-list sequences, each attached to two different random orders of the word pool.

\section{Subjects}

Ss were 34 undergraduate students from the University of Toronto. Eight or nine Ss were run on each set of lists, although no more than five Ss were run together.

\section{Procedure}

After a practice list of two digit numbers, Ss received 12 lists of words, with a 5-min break after the sixth list.

The lists were presented through a Sony video-tape system, with no more than three Ss viewing any one 11-in. monitor. Timing during taping was controlled by Hunter Timers set for a 2 -sec cycle. Visual words remained on for the whole 2-sec period.

Visual items were marked strictly, with allowances only for spelling mistakes. For auditory items, an additional "s" or "d" at the end of a word was accepted.

\section{RESULTS}

The results for repeated items are shown in Fig. 1. Clearly, different repetitions were not superior to same repetitions, even at short lags. A 34 by 2 by 2 by 4 ANOVA (Ss by Second Presentation Modality by Same/Different Repetition by Lag) showed a large lag effect, $F(3,99)=34.78, p<.001$, with recall increasing from $25.1 \%$ at $\operatorname{Lag} 0$ to $41.2 \%$ at $\operatorname{Lag} 9$. However, the effects of second presentation modality, $\mathrm{F}(1,33)<1.00$, same/different repetition, $F(1,33)<1.00$, and the interaction between second presentation modality and same/different repetition, $F(3,99)<1.00$, were significant.

The results for once-presented words are shown in Fig. 2. There were no modality differences in the middle of the list, a slightly greater primacy effect for visual items, and a stronger recency effect for auditory items. These results are similar to those from single-presentation mixed-modality experiments.

\section{DISCUSSION}

The failure to find any advantage for different repetitions, even at short lags, replicates Madigan and Doherty's (1971) results and also the main finding of a pilot study using 96-word lists. In the pilot study, visual items had an on-time of $0.5 \mathrm{sec}$ and an off-time of $1.5 \mathrm{sec}$, in contrast to the 2 -sec on-time in the present experiment. Hence, the on-off time for visual items does not appear to affect comparisons of repetitions within or across modalities.

Current theoretical explanations of the lag effect give no reason to expect that differences caused by spacing of repetitions and semantic variation should have an effect, whereas differences in modality do not. Two alternatives exist-either the current explanations of the lag effect are incorrect or they must be modified to distinguish between types of variation.

One possibility for an enconding variability explanation is to postulate that Ss organize lists on the basis of semantic groupings rather than modality groupings. Therefore, if adjacent words are presented in different languages or semantic contexts, then they may be associated with or rehearsed with different other words from the list. However, adjacent words in different modalities will be rehearsed in the same subjective unit. With spaced repetitions of words, each presentation of the word may be encoded within the context of the immediately adjacent words, so that the word occurs in at least two subjective units. Presumably, retrieval increases with the number of contexts in which an item is encoded. In consequence, spacing and semantic variation should increase recall, whereas physical variation such as modality should not. "A problem with this explanation is that Ss do cluster words by modality [Hintzman, Block, \& Inskeep, 1972]," so that even if Ss prefer to organize lists by semantic rather than modality groupings, one might still expect some effect from repetition in different modalities.

In summary, it is not sufficient merely to talk about variant repetitions; it is necessary to discuss the kind of variation

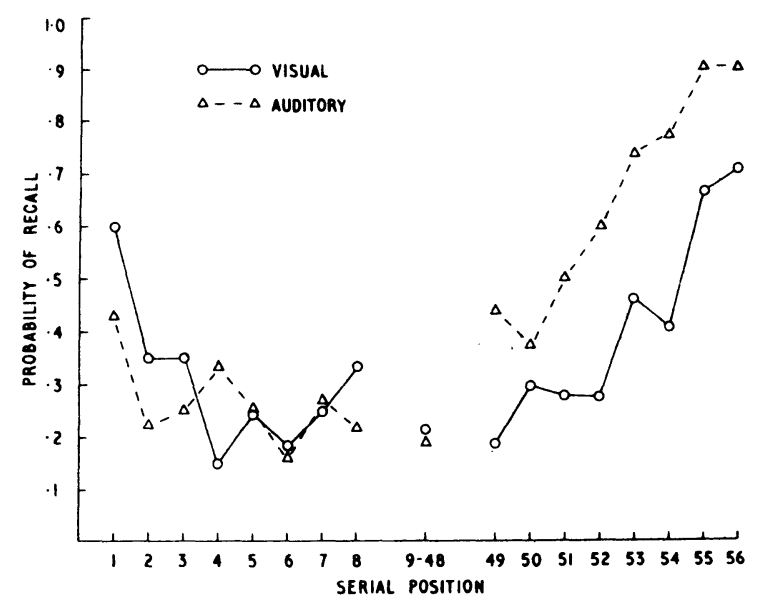

Fig. 2. Recall of single items (1P) as a function of serial position (102 observations per point except for Serial Positions 9-48, which are based on 1,632 observations). 
involved. Semantic or contextual variation improves recall, whereas modality variation does not. The work on release from PI (Hopkins, Edwards, \& Gravelek, 1971; Murdock \& Carey, 1972) also provides evidence that modality changes and semantic changes have different effects. Modality effects may be typical of those for physical variations or, alternatively, they may well be peculiar to modality alone.

\section{REFERENCES}

Bevan, W., \& Dukes, W. F. Stimulus-variation and recall: The role of belongingness. American Journal of Psychology, 1967, 80, 309-312.

Bjork, R. A., \& Allen, T. W. The spacing effect: Consolidation or differential encoding. Journal of Verbal Learning \& Verbal Behavior, 1970, 9, 567-572.

Bower, G. H. Stimulus-sampling theory of encoding variability. In E. Martin and A. W. Melton (Eds.), Coding processes in human memory. New York: Halsted Press, 1972.

D'Agostino, P. R., \& De Remer, P. Repetition effects as a function of rehearsal and encoding variability. Journal of Verbal Learning \& Verbal Behavior, 1973, 12, 108-113.

Glanzer, M., \& Duarte, A. Repetition between and within languages in free recall. Journal of Verbal Learning \& Verbal Behavior, 1971, 10, 625-630.
Hintzman, D. L., Block, R. A., \& Inskeep, N. R. Memory for mode of input. Journal of Verbal Learning \& Verbal Behavior, 1972, 11, 741-749.

Hopkins, R. H., Edwards, R. E., \& Gavelek, J. R. Presentation modality as an encoding variable in short-term memory. Journal of Experimental Psychology, 1971, 90, 319-325.

McCormack, P. D., \& Carboni, N. L. Lag invariance with forced encodings in free recall. Canadian Journal of Psychology, 1973, 27, 144-151.

Madigan, S. A. Intraserial repetition and coding processes in free recall. Journal of Verbal Learning \& Verbal Behavior, 1969, 8, 828-835.

Madigan, S., \& Doherty, L. Retention of item attributes in free recall. Psychonomic Science, 1972, 27, 233-235.

Melton, A. W. The situation with respect to the spacing of repetitions and memory. Journal of Verbal Learning \& Verbal Behavior, 1970, 9, 596-606.

Murdock, B. B., Jr., \& Carey, S. T. Release from interference in single-trial free recall. Journal of Verbal Learning \& Verbal Behavior, 1972, 11, 398-402.

Rundus, D. Analysis of rehearsal processes in free recall. Journal of Experimental Psychology, 1971, 89, 63-77.

(Received for publication January 18, 1974.)

\title{
Effects of shock intensity on speed and response competition in the escape training of neonatal and infant rats
}

\author{
JAMES R. MISANIN, SHERYL HARDY, and JANET GOODYEAR \\ Susquehanna University, Selinsgrove, Pennsylvania 17870 \\ and \\ Z. MICHAEL NAGY
Bowling Green State University, Bowling Green, Ohio 43402
}

\begin{abstract}
Rats, 3-11 days old, were given 25 escape training trials in a straight alley with .1, .2, or .4-mA $60-\mathrm{Hz}$ ac shock as the motivating stimulus. The results indicated that the younger the rat or the more intense the shock, the less likely escape training was to be completely effective. On .1 mA, only the 7 - to 11-day-old Ss showed an intrasession speed increase, and on .4 mA, only the 11-day-olds showed such an increase. The 7- to 11-day-old Ss were also the only Ss to increase speed as shock intensity increased. Response competition, on the other hand, decreased over training trials for all age groups on both .1 and $.2 \mathrm{~mA}$, but only the 5- and 7-day-old Ss showed such a decrease on .4 mA. For all age groups, however, response competition varied inversely with shock intensity.
\end{abstract}

A number of studies has suggested that turning is a reaction apparently high on the neonatal rat's response hierarchy when it encounters an aversive stimulus. Gard, Hard, Larsson, and Peterson (1967), for example, 